\title{
Feedback Control of the Sawtooth Period through Real Time Control of the Ion Cyclotron Resonance Frequency
}

\author{
M. Lennholm ${ }^{\mathrm{abc}}$, T. Blackman ${ }^{\mathrm{d}}$, I. T. Chapman ${ }^{\mathrm{d}}$, L-G. Eriksson ${ }^{\mathrm{ac}}$, J. P. Graves ${ }^{\mathrm{e}}$, \\ D.F. Howell ${ }^{\mathrm{d}}$, M. de Baar ${ }^{\mathrm{f}}$, G. Calabro ${ }^{\mathrm{g}}$, R. Dumont ${ }^{\mathrm{a}}$, M. Graham ${ }^{\mathrm{d}}$, S. Jachmich ${ }^{\mathrm{dh}}$, \\ M.L. Mayoral ${ }^{\mathrm{d}}$, C. Sozzi ${ }^{\mathrm{i}}$, M. Stamp ${ }^{\mathrm{d}}$, M. Tsalas ${ }^{\text {bkf }}$, P. de Vries ${ }^{\mathrm{dh}}$ \\ JET-EFDA, Culham Science Centre, Abingdon OX14 3DB, UK \\ ${ }^{a}$ CEA, IRFM, 13108 Saint-Paul-lez-Durance, France \\ ${ }^{b}$ EFDA Close Support Unit, Culham Science Centre, Abingdon OX14 3DB, UK \\ ${ }^{c}$ European Commission, B-1049 Brussels, Belgium \\ ${ }^{d}$ CCFE, Culham Science Centre, Abingdon OX14 3DB, UK \\ ${ }^{e}$ Association EURATOM-Confédération Suisse, Ecole Polytechnique Fédérale de Lausanne (EPFL), \\ CRPP, CH-1015 Lausanne, Switzerland \\ ${ }^{f}$ FOM Institute for Plasma Physics Rijnhuizen, Association EURATOM-FOM, Trilateral Euregio Cluster, \\ The Netherlands \\ ${ }^{g}$ Associazione EURATOM-ENEA sulla Fusione, C.R. Frascati, Roma, Italy \\ ${ }^{h}$ Max-Planck-IPP, Euratom Association, D-17491 Greifswald, Germany \\ ${ }^{i}$ Istituto di Fisica del Plasma CNR, Euratom Association, 20125 Milano, Italy \\ kAssociation EURATOM-Hellenic Republic, National Technical University of Athens, Iroon Politechniou 9, \\ 15773 Zografou, Athens, Greece
}

\section{Abstract}


Modification of the sawtooth period through Ion Cyclotron Heating and Current Drive (ICRF) has been demonstrated in a number of experiments. The effect has been seen to depend critically of the location of the ICRF absorption region with respect to the $q=1$ flux surface. Consequently, for ICRF to be a viable tool for sawtooth control one must be able to control the ICRF absorption location in real time so as to follow variations in the location of the q=1 surface. To achieve this, the JET ICRF system has been modified to allow the JET real time central controller to control the ICRF frequency. An algorithm for real time determination of the sawtooth period has been developed and a closed loop controller which modifies the ICRF frequency to bring the measured sawtooth period to the desired reference value has been implemented. The present paper shows the first experimental demonstration of closed loop sawtooth period control by real time variation of the ICRF frequency. 


\section{Introduction}

The MHD instability known as the sawtooth instability causes periodic crashes in the central plasma density and temperature [1]. This instability is associated with the flux surface at which the plasma safety factor $\mathrm{q}$ is equal to unity. The time between subsequent sawtooth crashes depends on a number of parameters and in JET this period varies over a wide range from a few milliseconds to several seconds. When the sawtooth period is large, the sawtooth crash has been seen to regularly trigger Neoclassical Tearing Modes (NTMs) [2,3]. These modes, located around flux surfaces where the safety factor takes on a rational value - typically 3/2 or 2/1 -, form a series of 'islands' around the rational flux surfaces $[4,5,6]$. These modes are stable for low normalised plasma pressures (beta). However if beta exceeds a certain threshold they become 'meta stable', which means that the modes grow if a sufficiently large 'seed island' is created by another event. The crashes associated with long period sawteeth regularly create such 'seed islands'. If, however, the sawtooth period is kept short the 'seed islands' are not large enough for NTMs to evolve [7]. NTMs degrade the plasma performance significantly and may even lead to disruptions; it is therefore highly desirable to avoid triggering these modes by maintaining short sawteeth.

The sawtooth period is seen to be significantly lengthened when fast ions are present in the plasma core $[8,9]$. The fast ions responsible for this stabilisation of the sawtooth instability can be NBI ions with energies in the $100 \mathrm{keV}$ range leading to a modest increase in the sawtooth period; ICRH generated $\mathrm{MeV}$ ions, potentially generating very long 'monster' sawteeth; or fusion generated alpha particles in deuterium 
tritium plasmas. In ITER and future reactors alpha particles are likely to generate very long sawteeth in the absence of control measures to shorten the sawtooth period.

According to the widely used model for the sawtooth crash of Ref [1], the equations governing the occurrence of a crash, under the conditions relevant for the experiments reported here, can be summarised as follows:

$$
\begin{aligned}
& S_{1}>S_{\text {crit }} \\
& S_{1}>\frac{C}{\hat{\rho}} \delta W
\end{aligned}
$$

For a crash to occur both (1) and (2) have to be fulfilled. In these equations $s_{1}$ is the shear $s=\frac{q}{r} \frac{d q}{d r}$ at the $\mathrm{q}=1$ surface, $\hat{\rho}$ is the normalised Larmor radius and $\delta W$ is the potential energy functional associated with the $m=1$ mode causing the sawtooth crash. The critical shear $s_{c r i t}$ and the parameter c are essentially determined by layer physics near the $\mathrm{q}=1$ surface.

Immediately following a sawtooth crash the central plasma current profile is flattened and either the q=1 surface disappears or $s_{1}$ becomes small. Subsequently $s_{1}$ grows as the central temperature and density increases until (5) is fulfilled and the next sawtooth crash occurs.

In the absence of fast ions a sawtooth crash will normally happen when_s $s_{1}$ reaches_ $s_{\text {crit }}$. The presence of fast ions in the plasma modifies $\delta W$ and with significant central fast ion pressures a sawtooth crash should not happen until $s_{1}$ has reached a level Field Code Changed where both the conditions in equations (1) and (2) are met, resulting in increased sawtooth period. Control of the sawtooth period can in this model be obtained by 
modifying either $s_{1}$ through local current drive or by modifying $\delta \mathrm{W}$. The influence of various heating and current drive systems on the sawtooth period have been reported from several tokamaks and this was recently reviewed in [11]. For instance the influence of Neutral beam injection has been reported from JET [12,13] while the effect of mode conversion current drive has been observed on Alcator C-mod [14]. Theoretical investigations of the effect of Electron Cyclotron Resonance Frequency (ECRF) Current Drive/Heating on sawteeth have been published by Angioni [15] while experimental investigations have been conducted on a number of tokamaks $[15,16,17,18,19]$. These investigations indicate that ECRF modify the sawtooth behaviour by affecting $s_{1}$ with little influence on $\delta W$. When on the other hand, ICRF is used for controlling the sawtooth period, both $s_{1}$ and $\delta W$ are affected and it is not easy to decouple the effect of modifying these two quantities. The effect of ICRF located near the $q=1$ surface was first observed on JET in the early 1990s [20] where a clear effect of the ICRF phasing was evident. Further JET experiments with $-90^{\circ}$ phased ICRF (in JET $-90^{\circ}$ relative phasing of the currents in adjacent straps, of the four strap antenna, gives rise to waves propagating predominately in the direction counter to the plasma current; and $+90^{\circ}$ phasing produces mostly co-current propagating waves) where the absorption region was moved from the plasma edge to the centre by varying the toroidal field and the plasma current together while keeping the $\mathrm{q}=1$ position constant have shown that a minimum sawtooth period is achieved with the ICRF location close to the $\mathrm{q}=1$ position and with long sawteeth being generated when the ICRF location reached the plasma centre [21]. More recent JET experiments using central ICRF with $+90^{\circ}$ phasing to create long sawteeth and $-90^{\circ}$ ICRF near $\mathrm{q}=1$ have demonstrated that localised ICRF can be used to shorten fast ion induced 
long sawteeth [22], thereby avoiding the triggering of NTMs [23]. The latter experiments showed an extreme sensitivity of the destabilising effect to the relative location of the Ion Cyclotron resonance with respect to the $\mathrm{q}=1$ surface. In fact a change of the distance between the $\mathrm{q}=1$ surface and the ion cyclotron resonance location of only a few $\mathrm{cm}$ resulted in a loss of the destabilising effect. Until recently the shortening of sawteeth achieved using ICRF near q=1 has been assumed to be achieved through local current drive and therefore through modification of $s_{1}$. It is, however, hard to explain the observed extreme sensitivity using this assumption. Recent theoretical work [24] has suggested an alternative explanation in which $\delta W$ is reduced by fast ions with specific passing orbits generated when the $-90^{\circ}$ ICRF is absorbed in a narrow radial region. This fast ion orbit explanation has been tested in experiments using ${ }^{3} \mathrm{He}$ minority ICRF in which the driven current should have been kept very low. The results provided evidence in favour of the fast ion orbit explanation [25]. Regardless of the mechanism responsible for the sawtooth destabilisation, the observed extreme sensitivity means that controlling the ICRF absorption location in real time is essential for ICRF to be a viable tool for sawtooth control on future machines.

Even for the use of ECRF for sawtooth control, where the destabilisation seems to be clearly achieved through a modification of $s_{1}$, real time control of the power deposition is required. Feedback control of the sawtooth period using ECRF to modify $s_{1}$ has first been demonstrated in the presence of ICRF generated fast ions on Tore Supra $[10,26]$ and later without fast ions on TCV [27]. When ECRF is used for sawtooth control the location of the ECRF absorption is moved by steering the injected ECRF beam via mobile mirrors situated inside the tokamak. For ICRF, the only way to move 
the absorption position without modifying fundamental plasma parameters, such as toroidal field, plasma position or plasma current, is to change the ion cyclotron resonance position relative to the $\mathrm{q}=1$ surface by changing the ICRF frequency. This paper describes the first demonstration of the use of real time variation of the ICRF frequency for feedback control of the sawtooth period, as would be required if ICRH is to be used as a robust control actuator in ITER.

\section{Control loop implementation}

Figure 1 shows the closed loop control scheme in a very simplified way. The control algorithm is implemented in the JET Real Time Central Controller (RTCC) $[28,29]$. This controller is a universal controller which receives data from a number of JET diagnostics in real time. Control algorithms can be programmed into RTCC using a high level interface allowing the implementation of complex control block diagrams. The central controller can be given control of various actuators such as heating powers, phases etc. For the purpose of implementing feedback control of the sawtooth period, RTCC has to be able to take control of the ICRF frequency and it has to receive sufficient diagnostic information to be able to determine the sawtooth period in real time.

\section{Real time control of ICRH/CD frequency}

The first step to implement the control loop was to assure that RTCC could take control of the ICRF frequency. This required minor hardware changes in the ICRF plant. Prior to the implementation of these changes, the ICRH frequency was controlled internally by the ICRF plant [30]. The frequency could be varied in real time and this 
ability was used to assist the matching of the ICRF generators to the load presented to them by the ensemble of transmission lines, antenna and plasma. Matching of the system is achieved on a slow timescale using mechanical matching elements such as trombones and stubs. These elements move rather slowly and frequency variation was employed to allow matching in the case of fast variations in the plasma loading of the ICRF antennae. It was, however, found that the use of frequency variation for matching purposes was often not required, which left the frequency variation free to be used for other purposes. The range over which the frequency can be varied in real time is limited to $+/-500 \mathrm{KHz}$ around a central value. This limitation has two origins: Firstly, the JET ICRF system can only operate in a series of bands of a few $\mathrm{MHz}$ between $23 \mathrm{MHz}$ and $57 \mathrm{MHz}$ [30]. Secondly, just as ICRF frequency variation can be used for matching purposes, a change of the frequency, when the system is well matched, will result in a mismatch. This mismatch has to be recovered by moving the mechanical matching elements - more specifically the trombones - and the range of variation of the length of these trombones $(0-1.5 \mathrm{~m})$ puts an upper limit to the maximum frequency variation over which matching can be maintained. The maximum of $+/-500 \mathrm{KHz}$ could potentially be increased slightly but not much before one of the mentioned hard limits are encountered. The fact that the trombones have to move to maintain matching when the frequency changes strongly limits the rate at which the frequency can be changed. The installation of $3 \mathrm{~dB}$ couplers in the ICRF transmission lines which directs reflected power into dummy loads rather than back to the RF generators allows the system to work in less well matched conditions [31]. Due to these $3 \mathrm{~dB}$ couplers, modest frequency variations $<50 \mathrm{kHz}$ can be made without moving the trombones and hence fine tuning can be done rapidly while larger changes 
have to be made much more slowly. An additional problem to be considered when implementing the control algorithm in RTCC is that the speed of movement of the trombones is controlled by the internal ICRF matching system and this speed is proportional to the observed mismatch. In order to change the position of the trombone as rapidly as possible, the mismatch has to be as large as possible. For the quickest response the requested frequency has to be as far from the matched point as possible without the matching becoming so poor that the ICRH generator trips out. The $3 \mathrm{~dB}$ couplers mentioned above are essential to allow a sufficiently large mismatch for the trombones to be made to move with reasonable speed. Unfortunately $3 \mathrm{~dB}$ couplers are only installed on half of the JET antennae and therefore the ICRF power for which real time control of the frequency is available is limited to about 5MW. Details of how the controller copes with this indirect control of the trombone movement are presented later.

To allow RTCC to take control of the frequency of the ICRF system, the wiring of the plant was modified with the frequency control input previously used by the internal matching control being connected to an output from the Radio Frequency Local Manager (RFLM) which is a unit situated near the ICRF plant, responsible for the communication between RTCC and the ICRF system. The final step to allow the control was to implement software changes in RTCC and RFLM creating an RTCC 'ICRF frequency deviation' output and an RFLM 'frequency deviation' input and transmitting this quantity across the ATM communication network linking RTCC and RFLM. 


\section{Real Time Sawtooth Period Determination}

The Real Time determination of the sawtooth period in the present experiment was achieved by a simple algorithm implemented in RTCC. The determination was based on signals from the Electron Cyclotron Emission (ECE) diagnostic [32]. In this diagnostic which measures the electron temperature at 96 values of the major radius, periodic sawtooth 'crashes' appear as a rapid reduction in the electron temperature for central channels (normal sawteeth) and as a simultaneous rapid increase of the temperature in external channels (inverted sawteeth). Following a sawtooth 'crash' the central temperatures increases and the outer temperatures decrease until the subsequent crash occurs. The radius inside which normal sawteeth are observed and outside which inverted sawteeth are seen, the sawtooth inversion radius, is closely related to the $\mathrm{q}=1$ surface. Two ECE real time signals were available for use by RTCC - a central temperature and an average over a selected range of major radii. Prior to a pulse the ECE channels used to produce these real time signals can be chosen. In the experiments presented in this paper an average over off axis channels - showing inverted sawteeth proved most robust in reliably detecting sawtooth crashes without too many false detections. The cycle time of RTCC is $10 \mathrm{~ms}$ meaning that the real time signals are only acquired by RTCC every 10ms which is much slower than the ECE data acquisition. This did not pose a problem for detecting sawteeth as long as the sawtooth period did not go below $\sim 20 \mathrm{~ms}$. In the present series of experiments the sawtooth period remained above this level as determined by post pulse analysis using the full resolution of the ECE diagnostic. As a matter of fact the slow acquisition actually made it easier to implement a 
simple sawtooth crash detection algorithm as sawtooth crashes were always manifest through a large temperature jump from one time sample to the next. A simple threshold on $\Delta T_{e}(n)=T e_{a v}(n)-T e_{a v}(n-1)$ was therefore sufficient to determine when a sawtooth crass had occurred. $T e_{a v}(n)$ is the average electron temperature as measured by a number of off axis ECE channels for the $n$th time point. Having detected a sawtooth crash, the time of this crash $t_{c 0}$ is memorised. When the subsequent crash is detected at the time $t_{c 1}$ the period of the last sawtooth is determined as $\Delta t_{0}=t_{c 1}-t_{c 0}$. Having determined $\Delta t_{0}$, $t_{c 0}$ is updated by setting $t_{c 0}=t_{c 1}$. Between sawtooth crashes the real time value of the sawtooth period is determined as the maximum of the time passed since the last sawtooth crash and the length of the previous sawtooth $\Delta t=\max \left(t-t_{c 0}, \Delta t_{0}\right)$.

Figure 2 shows the real time sawtooth period determination. In the top box the sawteeth as observed with the ECE diagnostic at three different major radii are shown. One signal - red trace - originates near the plasma centre (Major Radius $\mathrm{R}=2.93 \mathrm{~m}$ ) and shows clear 'normal' sawteeth, one signal - blue trace - originating from a larger radius $(\mathrm{R}=3.24 \mathrm{~m})$ show clear 'inverted' sawteeth while the sawteeth are hardly visible in the third signal - magenta trace - originating at an intermediate position $(\mathrm{R}=3.17 \mathrm{~m})$. The latter signal indicates the position of the 'sawtooth inversion radius' close to the $q=1$ magnetic surface. The second box in figure 2 shows the inverted sawteeth as observed by RTCC with a 10ms sampling time while the third box shows the time evolution of $\Delta T_{e}$ and the threshold for sawtooth crash detection. The final box shows the real time sawtooth period $\Delta t$ (blue trace) and the time since the last sawtooth $t-t_{0}$ (red trace) together with the sawtooth period determined post pulse using the full ECE resolution 
(green dotted trace). The real time sawtooth period which always takes a value which is an integer multiple of the sample time $(10 \mathrm{~ms})$ is seen to underestimate the sawtooth period by an average of $10 \mathrm{~ms}$. As this is a constant offset, it has no impact on the use of this signal for real time control purposes.

For a more universal Real Time sawtooth period detection an algorithm of the type described in [26] should be implemented. Such an algorithm would be hosted in the ECE diagnostic itself and it would provide real time sawtooth period and inversion radius for RTCC to use.

\section{Controller}

The controller implemented is fundamentally a simple Proportional Integral

Derivative (PID) controller which takes as input the sawtooth period error:

$\Delta t_{\text {err }}=\Delta t_{\text {ref }}-\Delta t$, where $\Delta t_{\text {ref }}$ is the sawtooth period reference, and produces the required deviation $\Delta f$ of the RF frequency from its centre value as an output. This frequency deviation is then sent to the RFLM where it is added to the centre frequency $f_{0}$ to get the RF frequency request $f=f_{0}+\Delta f$ used internally by the ICRH plant. As mentioned earlier, a certain complication is added to this basic control algorithm due to the slow response of the ICRH matching trombones. If the value of $\Delta f$ varies too much too quickly, the RF plant will not be well matched to the 'plasma - antenna transmission line' assembly and as a consequence the RF power will be cut off due to excessive reflected power seen by the plant. To assure that the controller does not request frequencies that would make the RF plant trip out, the controller should know the actual 
position (length) of the matching trombones. This position can then be translated into a frequency at which perfect matching is achieved:

$$
f_{\text {match }}=f(\Delta L) \cong f_{0}+k_{T} \cdot \Delta L,
$$

In this equation $k_{T}$ is a constant and $\Delta L=L-L_{0}$, where $L$ is the current trombone position and $L_{0}$ is the trombone position that achieves perfect match when $f=f_{0}$, Subtracting $f_{0}$ the value of $\Delta f$ which achieves perfect matching can be introduced:

$$
\Delta f_{\text {match }} \cong k_{T} \cdot \Delta L
$$

Based on the knowledge of the frequency corresponding to perfect matching, limits can be introduced preventing the controller from requesting frequencies which deviate more than a certain value $\Delta f_{\max \text { mismatch }}$ from the matched frequency:

$$
\begin{aligned}
& \Delta f_{\text {max }}=\Delta f_{\text {match }}+\Delta f_{\text {max mismatch }}=k_{T} \cdot \Delta L+\Delta f_{\text {max mismatch }} \\
& \Delta f_{\text {min }}=\Delta f_{\text {match }}-\Delta f_{\text {max mismatch }}=k_{T} \cdot \Delta L-\Delta f_{\text {max mismatch }}
\end{aligned}
$$

This way $\Delta f$ is forced to remain within a band around the - varying - matched value $\Delta f_{\text {match }}$. This would be easy to implement if the position of the trombone was known by the controller. Unfortunately the trombone position is an internal signal within the ICRH plant and it is not available for use by the controller. To overcome this problem a model of the behaviour of the trombone controller has been built into the RTCC controller. The trombone controller uses a simple proportional controller where the trombone velocity is proportional to the observed mismatch. The mismatch used by the trombone controller can be modelled as the deviation of the actual RF frequency from the frequency at which perfect matching is obtained: 


$$
\Delta f_{\text {mismatch }}=f_{0}+\Delta f-f_{\text {match }}=\Delta f-\Delta f_{\text {match }}
$$

And hence the requested trombone velocity can be simulated as:

$$
V_{\text {sim_ref }}=k_{V}\left(\Delta f-\Delta f_{\text {match }}\right)
$$

To take into account the inertia of the trombone and the maximum speed with which the trombone can move, the simulated trombone velocity is calculated as a filtered version of $V_{\text {sim_ref }}$ limited by the maximum trombone speed $V_{\text {sim_max }}>0$ :

$$
V_{\text {sim }}=\text { filt }\left(V_{\text {sim_ref }_{\text {ref }}}\right) \text { and }\left|V_{\text {sim }}\right|=\leq V_{\text {sim_max }}
$$

Finally the trombone position $L_{\text {sim }}$ is simulated - assuming that the initial position is the position corresponding to matching with $\Delta f=0$ - by integrating the $V_{\text {sim }}$ signal:

$$
L_{\text {sim }}=\int_{0}^{t} V_{\text {sim }}+L_{0}
$$

Having determined the limits to $\Delta f$ imposed by the slow trombone movement two additional limits are also imposed. Firstly the 'slew rate'- or in plain English, the maximum variation of the requested frequency from one time point to the next - is limited to $10 \mathrm{kHz}$ and finally the maximum value of $\Delta f$ is limited to slightly less than the $500 \mathrm{kHz}$ that the ICRF plant can accept.

Figure 3 shows how the controller including the trombone simulator and associated limits is implemented as control blocks in the RTCC high level controller language.

Figure 4 shows the behaviour of the trombone simulator and how the value of $\Delta f$ is limited as a consequence. It should be noted that throughout most of the shown discharge the unlimited $\Delta f$ request did not exceed the trombone limits by a large 
amount. This was due to a combination of a modest proportional gain in the PID controller, a small value of the error signal input to the controller and the filtering of $\Delta f$ provided through the integral term in the PID controller.

To be able to optimise the controller settings, a simple 'plasma simulation' was implemented in the RTCC. This simulation did not attempt to simulate sawteeth but simulated only the sawtooth period based on the value of the $\Delta f$ signal and assuming a simple transfer function derived from the open loop experiments described in the following. This very simple model allowed a reasonable tuning of the gains used in the PID controller with the result that no further tuning proved necessary when closed loop experiments were attempted.

\section{Experiments}

\section{Open Loop}

Before proceeding to closed loop operation, open loop experiments were performed. As the range of variation of the RF frequency is limited, varying this frequency allows only a modest variation of the position of the Ion Cyclotron resonance. For this reason it was decided to repeat the open loop experiments of [21] scanning toroidal field and current over a wide range while keeping the position of $\mathrm{q}=1$ constant.

Figure 5 shows two consecutive discharges where such a scan was performed with the resonance moving from the edge to the plasma centre in \#68273 and from the centre to the edge in \#68275. The figure shows how the sawtooth period has a general trend increasing when the resonance location moves from the edge to the centre. The more noteworthy observation is, however, the fact that this trend is broken in a narrow 
range when the major radius of the Ion Cyclotron resonance, is slightly closer to the plasma centre than the major radius of the $\mathrm{q}=1$ surface at the plasma mid plane as determined EFIT from magnetic measurements. In this connection the absolute location of $\mathrm{q}=1$ should be taken with some caution as no precise current profile measurement is available. It should, however, be noted that the measured sawtooth inversion radius is well correlated with the EFIT $\mathrm{q}=1$ radius and that neither change significantly throughout the scan.

Figure 6a shows the sawtooth period as a function of the major radius location of the ICRF resonance for the two discharges shown in figure 5. It is seen that the two discharges give almost identical results with the traces shifted slightly towards the left (high field side) for the discharge where the resonance was scanned from the centre towards the edge.

Figure $6 \mathrm{~b}$ shows the same discharges with the sawtooth period plotted as a function of the distance between the major radius of the ICRF resonance and the location of the $\mathrm{q}=1$ surface at the mid-plane inferred from EFIT. The two discharges now overlap perfectly, showing that the shift seen in figure 6a was due to a change in the location of the $\mathrm{q}=1$ surface. This change in the $\mathrm{q}$ profile is understandable due to the central plasma temperature being higher following the initial central heating in the second discharge.

The perfect overlap observed in figure $6 \mathrm{~b}$ is remarkable (and convenient from a control point of view) showing that scanning in opposite directions gives exactly the same results and hence there is no hysteresis in the response of the sawtooth period to variations in Ion Cyclotron deposition location other than those associated with evolution in the q profile. Figure $6 \mathrm{c}$ shows a zoom to the narrow range just inside the $\mathrm{q}=1$ surface 
where sawteeth shorten when the Ion Cyclotron absorption location is moved towards the centre. The shaded area shows the range of variation over which the absorption can be moved by varying the ICRF frequency over its full $+/-500 \mathrm{KHz}$ range while keeping field and current constant at the value corresponding to the centre of the range. It is seen that $+/-500 \mathrm{KHz}$ is (just) enough to allow significant variation of the sawtooth period.

In pulses with significant central fast ion pressure it has, as mentioned in the introduction, been observed that a larger and very abrupt change in sawtooth period is achieved when the location of destabilising off axis ICRF is changed by a much smaller amount than the one corresponding to the above range. This would mean that in this case the available ICRF frequency variation would be largely sufficient to cover the transition region. To simplify the experiments for an initial demonstration of feedback control using the ICRF frequency variation technique, it was decided to start without fast ions in the plasma centre. Therefore the transfer function shown in figure 6 was used to set the controller gains. As the available frequency range corresponds only to the monotonic part of the transfer function shown in the enlarged part of figure 6, the non-monotonic character of the complete transfer function can be ignored when setting up the controller and it can be assumed that the sawtooth period is reduced when the Ion Cyclotron resonance moves towards the plasma centre. Due to the positioning of the Ion Cyclotron Resonance on the High field side of the magnetic axis a movement towards the centre requires a decrease of the ICRF frequency. 


\section{Closed Loop}

Once the controller parameters had been selected a series of closed loop discharges were run. One of these discharges is shown in figure 7 . The requested sawtooth frequency is set to $30 \mathrm{~ms}$ and the control loop is activated at $10.5 \mathrm{~s}$ after which the frequency is varied in closed loop to bring the measured sawtooth period to the requested value. As can be seen, the requested sawtooth period is reached after less than a second. After the initial adjustment phase the sawtooth period is maintained close to $30 \mathrm{~ms}$ for the duration of the ICRH pulse. It is interesting to note that, to maintain the 30ms period, the controller has to continue to move the resonance towards the plasma centre by decreasing the ICRH frequency. This shows that the loop is actually capable of responding to changes in the plasma parameters. A few 70-80ms sawteeth observed on the real time signal are due to failure of the detection algorithm to detect a sawtooth crash. It is obviously difficult to set the crash detection level for the very small sawteeth achieved in this discharge and a more sophisticated algorithm could be beneficial to

improve the detection reliability. Nevertheless only very few crashes were missed and this problem did not adversely affect the behaviour of the controller. To verify that this control was not achieved by chance, the discharge was repeated with the only change being that the requested sawtooth periods was changed to $80 \mathrm{~ms}$. Figure 8 show this discharge together with the previous discharge. For the second discharge the controller continuously increased the ICRF frequency in order to move the resonance further from the plasma centre. As desired the sawtooth period increased towards the requested $80 \mathrm{~ms}$ though it saturated at $60 \mathrm{~ms}$. Thus, the control observed in \#76003 was not obtained by 
chance and despite the saturation at $60 \mathrm{~ms}$ these two pulses demonstrate a successful closed loop control of the sawtooth period within the achievable range of $30-60 \mathrm{~ms}$.

A legitimate query is whether the changes in observed sawtooth period could have been more due to the small power variations than due to the variation of the Ion Cyclotron resonance location. In order to address this issue, Figure 9 shows data for four consecutive pulses including the above discharges. In figure 9a the sawtooth period - as measured post pulse using high resolutions data - is plotted as a function of the distance between the plasma centre and the ICRF resonance position. In figure 9b the sawtooth period is plotted as a function of the injected power. Figure 9a clearly shows that the sawtooth period varies with the resonance location whereas figure $9 \mathrm{~b}$ is just a cloud of points showing that the small variations in power level have very little, if any, influence on the sawtooth period. It should be recalled that the real time estimate of the sawtooth period is in general underestimated by $10 \mathrm{~ms}$ - or $1 \mathrm{RTCC}$ cycle time which is the reason that the sawtooth periods seen in figure 9 range from $40 \mathrm{~ms}$ to $70 \mathrm{~ms}$ rather than from $30 \mathrm{~ms}$ to $60 \mathrm{~ms}$ as seen in Figure 8.

\section{Future Work.}

The experiments described above have demonstrated that the sawtooth period can be controlled by a feedback loop using the ICRF frequency as the actuator. These discharges have also shown the limitations caused by the slow trombone speed and by the modest range over which the ICRF frequency can be changed in real time. These limitations will be less problematic in more relevant discharges with fast ions in the plasma centre. In such discharges much larger changes in the sawtooth period are 
observed when destabilising ICRF is applied near the $q=1$ surface and these changes occur for smaller variations in the ICRF absorption location. The experiments described in this paper did not use central ICRF to generate fast ions in the plasma centre in order maintain the experiments as simple as possible. The wide scan of ICRF resonance position in the open loop experiments could not be performed in conjunction with central ICRF as the central resonance would move as much as the off axis ICRF resonance. As a consequence it was decided to initially demonstrate the real time control under the same conditions as the open loop discharges, thus allowing the controller parameters to be set up based on the results of the open loop experiments. Future experiments should focus on discharges in which a central fast ion pressure is created either by central ICRF or by NBI. These are also the type of discharges where sawtooth control will be required and where real time fine tuning of the resonance position will be essential to achieve such sawtooth control. Despite the faster variation of the sawtooth period in this case it would still be desirable to run longer discharges to allow the control loop to prove its capabilities over times significantly in excess of the control loop time constants. On JET the maximum pulse length of the ICRF system of $20 \mathrm{~s}$ should be sufficient to test the dynamic behaviour of the controller in future experiments.

\section{Conclusions}

Closed loop feedback control of the sawtooth period has been demonstrated in JET. For the first time such closed loop control has been achieved with ICRF, moving the ICRF resonance position by changing the ICRF frequency in real time. These experiments are promising in view of the potential for using this technique for sawtooth 
control on ITER as a complement to the use of ECRF as currently planned [33]. The fact that the ICRF control mechanism is more likely to be a fast ion effect $[24,25]$ would mean that ICRF sawtooth control could be more attractive in ITER where shear modifications through ECRF current drive may prove less effective than on current tokamaks, especially in the absence of fast ion control. The experiments also highlight that it would be desirable to design an ITER ICRF system which allows variation of the ICRH frequency in real time over a wide enough range to allow the resonance position to be moved over a larger part of the plasma radius than is possible at JET with the present ICRH system. It should be noted that the current ITER ICRF system design would allow

${ }^{3} \mathrm{He}$ minority ICRF to be absorbed near the q=1 surface with rapid frequency variation available to allow adjustment of the absorption range within a $20 \mathrm{~cm}$ range [34]. The slow response seen in the present experiments does not necessarily pose a problem on ITER where time constants are much longer than on JET. Further proof of this technique in more relevant plasma conditions with significant fast ion pressures in the plasma centre are required to demonstrate that this is a viable tool for sawtooth control and NTM avoidance on ITER.

\section{Acknowledgments}

This work was supported by EURATOM and carried out within the framework of the European Fusion Development Agreement. The views and opinions expressed herein do not necessarily reflect those of the European Commission. 


\section{References}

[1]F. Porcelli, D. Boucher and M.N. Rosenbluth, Plasma Phys. Control. Fusion 38, 2163

(1996).

[2]O. Sauter et al., Phys. Rev. Lett. 88, 105001 (2002).

[3] I. T Chapman et al., Nucl. Fusion 50, 102001 (2010).

[4]O. Sauter et al., Phys. Plasmas 4, 1654 (1997).

[5]R.J. Buttery et al., Plasma Phys. Control. Fusion 42, B61 (2000).

[6]R.J. La Haye et al., Phys Plasmas 9, 2051 (2002).

[7]E. Westerhof et al., Nucl. Fusion 42, 1324 (2002).

[8]D.J. Campbell et al., Phys. Rev. Lett. 60, 2148(1988).

[9]C.K. Philips et al., Phys. Fluids B 4, 2155 (1992).

[10]M. Lennholm et al., Phys. Rev. Lett. 102, 115004 (2009)

[11]I.T. Chapman. Sumitted to Plasma Phys. Control. Fusion

[12] C. Angioni et al., Plasma Phys. Control. Fusion 44, 205 (2002).

[13] I.T. Chapman et al. Plasma Phys. Control. Fusion 50, 045006 (2008).

[14] S.J. Wukitch et al., Physics of Plasmas 12, 056104 (2005)

[15]C. Angioni, T.P. Goodman, M.A. Henderson and O. Sauter, Nucl. Fusion 43, 455

(2003).

[16]A.Mueck et al., Plasma Phys. Control. Fusion 47, 1633 (2005).

[17]Y. Ikeda et al., Nucl. Fusion 42, 375 (2002).

[18] Z.Shi et al., Plasma Science and Technolog, vol.9 No.5, 534 (2007).

[19]T. Hender et al., Nucl. Fusion 47, S128 (2007).

[20]V.P. Bhatnagar et al., Nucl. Fusion 34, 1579 (1994). 
[21] M-L. Mayoral et al., Phys. Plasmas 11, 2607 (2004);

[22]L.G. Eriksson et al.,Phys.Rev.Lett. 92, 235004(2004)

[23]S. Coda et al Proc. 34 ${ }^{\text {th }}$ EPS Warsaw ECA Vol. 31F, P-5.130 (2007)

[24]J. Graves et al., Phys. Rev. Lett. 102, 065005 (2009)

[25]J. Graves et al., Nucl. Fusion 50052002 (2010)

[26] M. Lennholm et al., Fusion Sci. And Tech. 55, 45 (2009)

[27] J. I. Paley et al., Plasma Phys. Control. Fusion 51124041 (2009)

[28] M. Lennholm et al., Fusion Engineering and Design 48, 37 (2000)

[29] Q. King and H. Brelen, JET preprints: JET-P(98)24

[30] T. J. Wade, A. Kaye, J. Jaquinot, Proc. 7th SOFT, Rome, 1986 (Elsevier, Amsterdam, 1987) pp. 1398-1403.

[31]I. Monakhov et al., AIP Conf. Proc. 1187205 (2009)

[32] E. de la Luna et. al., Rev. Sci. Instrum. 75 (10) 3831 (2004)

[33] C. Zucca et al. Varenna - Lausanne Theory Conference, 1069, p361 (2008)

[34] P. Lamalle. Private communication 


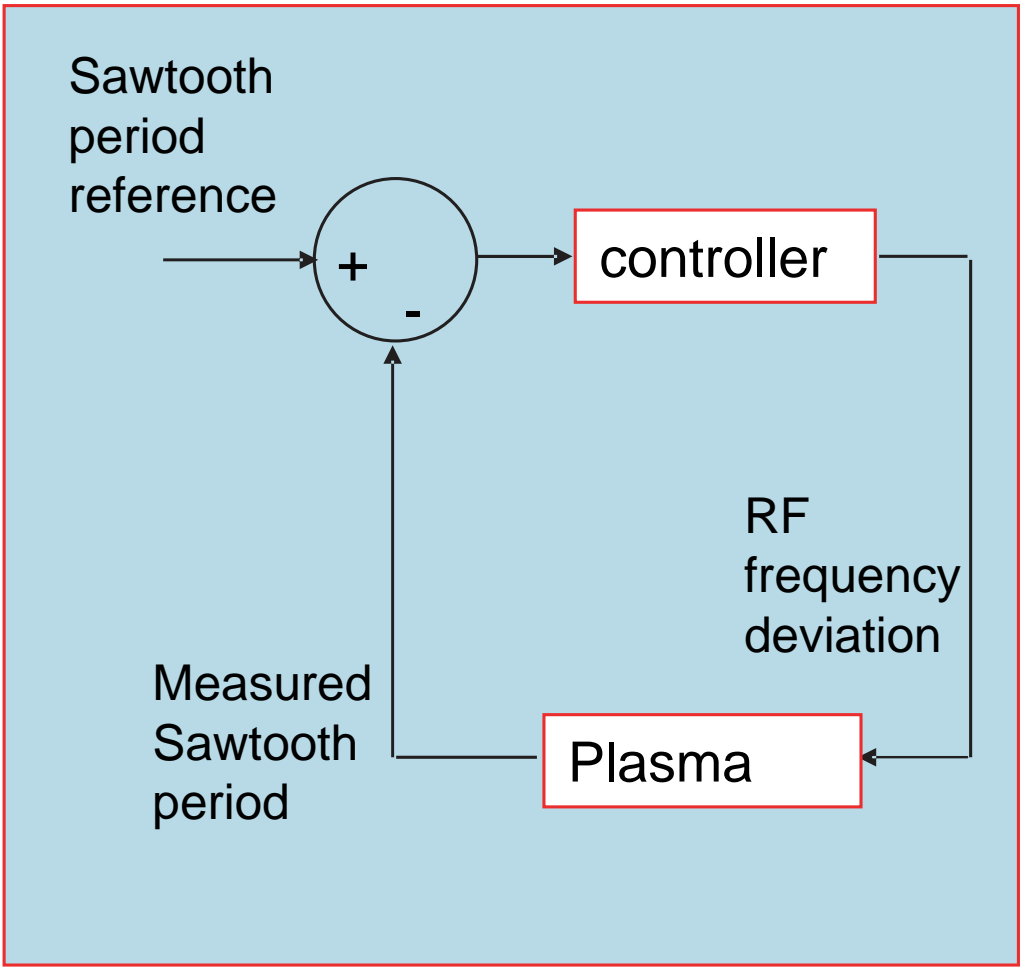

Figure 1 - Basic sawtooth period controller concept 


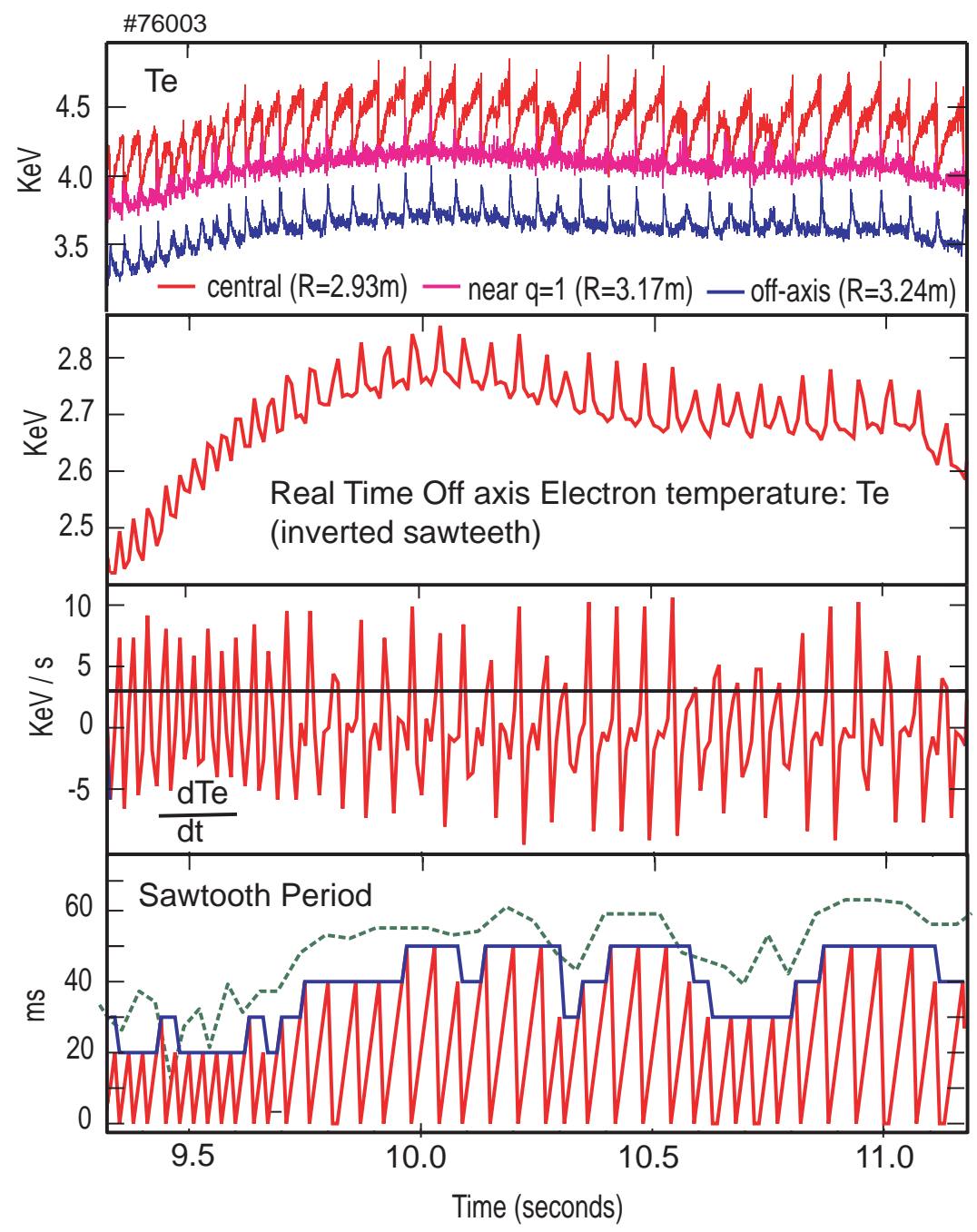

Figure 2 Real Time Sawtooth period determination. $1^{\text {st }}$ box: Data from Electron Cyclotron Emission diagnostic showing electron temperatures in the plasma centre(red), near the $\mathrm{q}=1$ surface(magenta) and in the outer part of the plasma(blue). $2^{\text {nd }}$ box: Real time off axis temperature signal used by RTCC. $3^{\text {rd }}$ box: Time derivative of real time temperature signal(red) and threshold for sawtooth detection(black). $4^{\text {th }}$ box: Real time sawtooth period(blue), time since last sawtooth(red) and sawtooth period determined post pulse(dashed-green). 


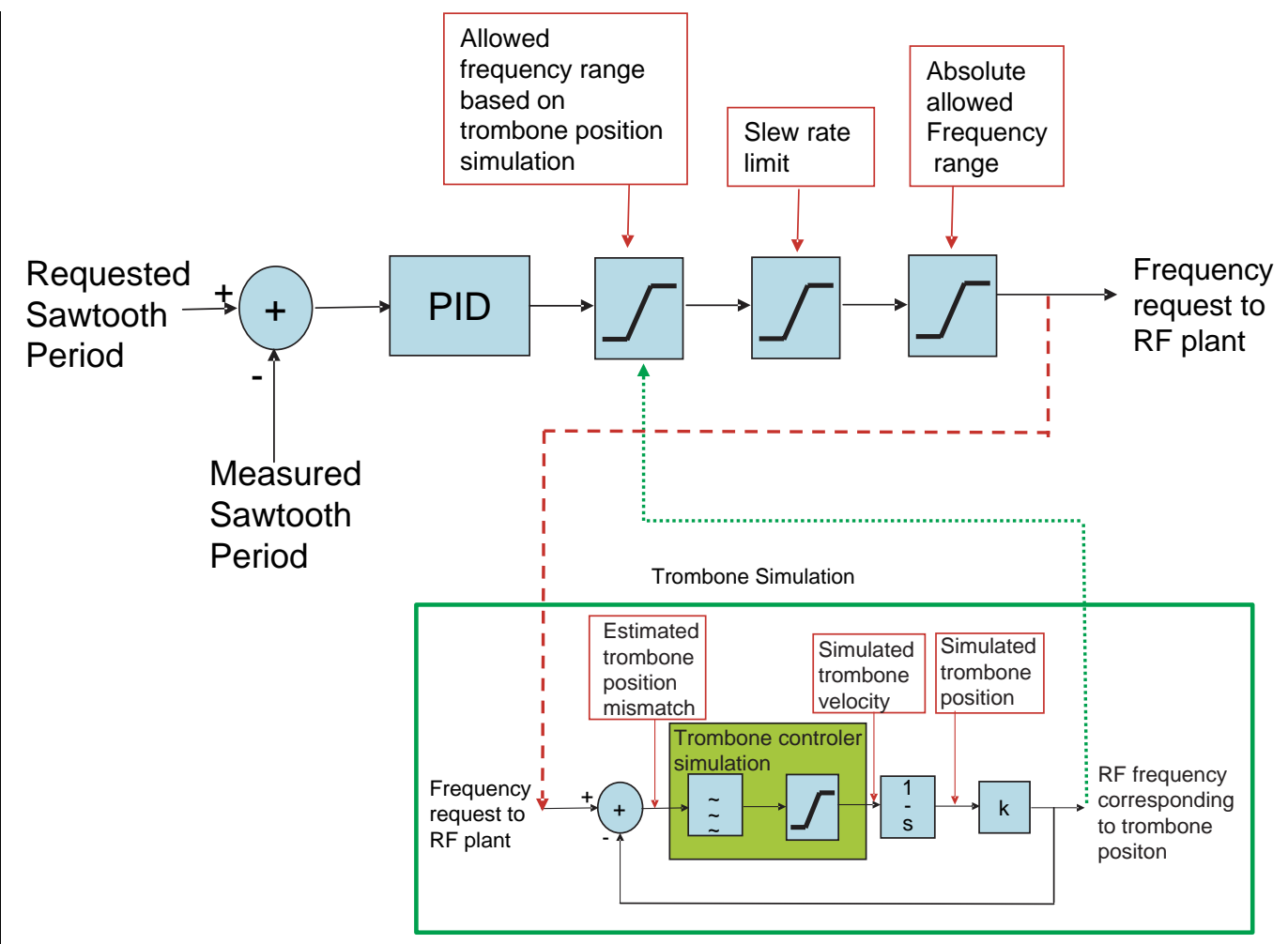

Fig 3 - Block diagram showing the way the ICRF frequency controller is implemented in

RTCC. The bottom box shows the real time trombone position simulation. 


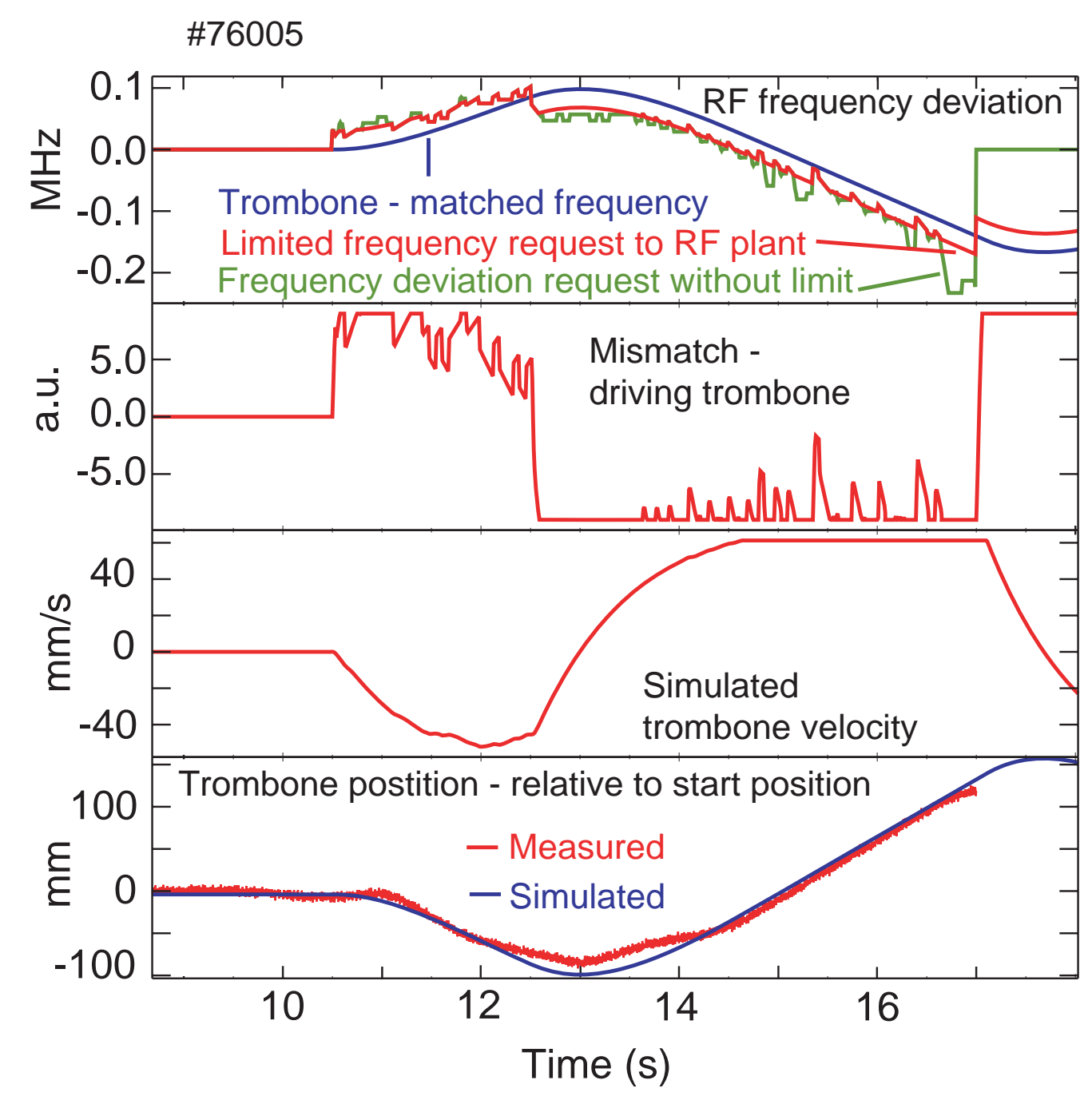

Fig 4. Trombone position simulation: $1^{\text {st }}$ Box: Frequency corresponding to perfect match with current simulated trombone position (blue), Unlimited frequency deviation request (green), Limited frequency deviation request transmitted to ICRF plant. $2^{\text {nd }}$ Box:

simulated mismatch driving the trombone movement. $3^{\text {rd }}$ box: Simulated trombone velocity. $4^{\text {th }}$ Box: Simulated and measured Trombone position . 


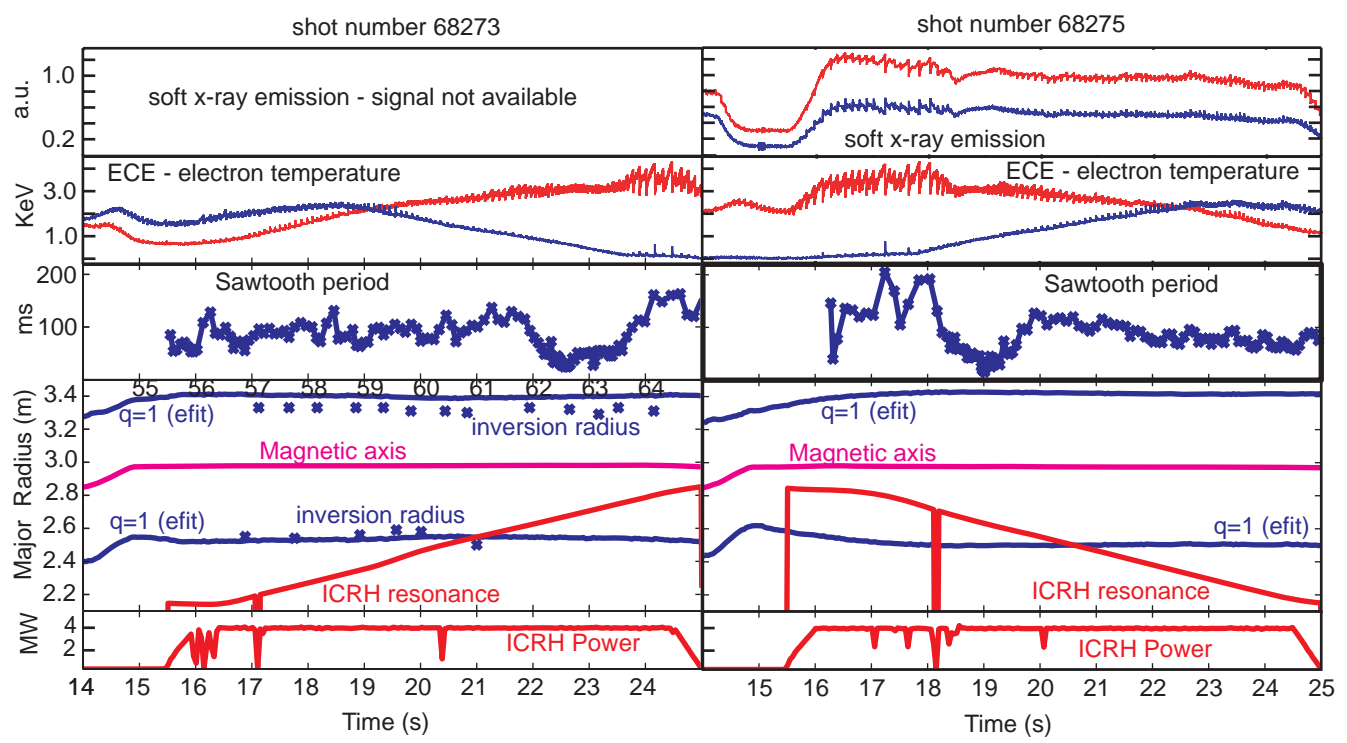

Figure 5: Two discharges where the magnetic field and plasma current were scanned simultaneously, while keeping the position of the $\mathrm{q}=1$ surface constant. $\quad 1^{\text {st }}$ row: Soft X-ray signals showing normal and inverted sawteeth (only available for \#68275). $2^{\text {nd }}$ row: ECE electron temperatures from two channels. The radial location of these signals varies as the magnetic field is scanned. $3^{\text {rd }}$ row: Sawtooth period - determined post pulse. $4^{\text {th }}$ row: Positions of ICRF resonance(red), $q=1$ (EFIT) (blue traces), sawtooth inversion radius (blue crosses) and magnetic axis (magenta). $4^{\text {th }}$ row: ICRF power 

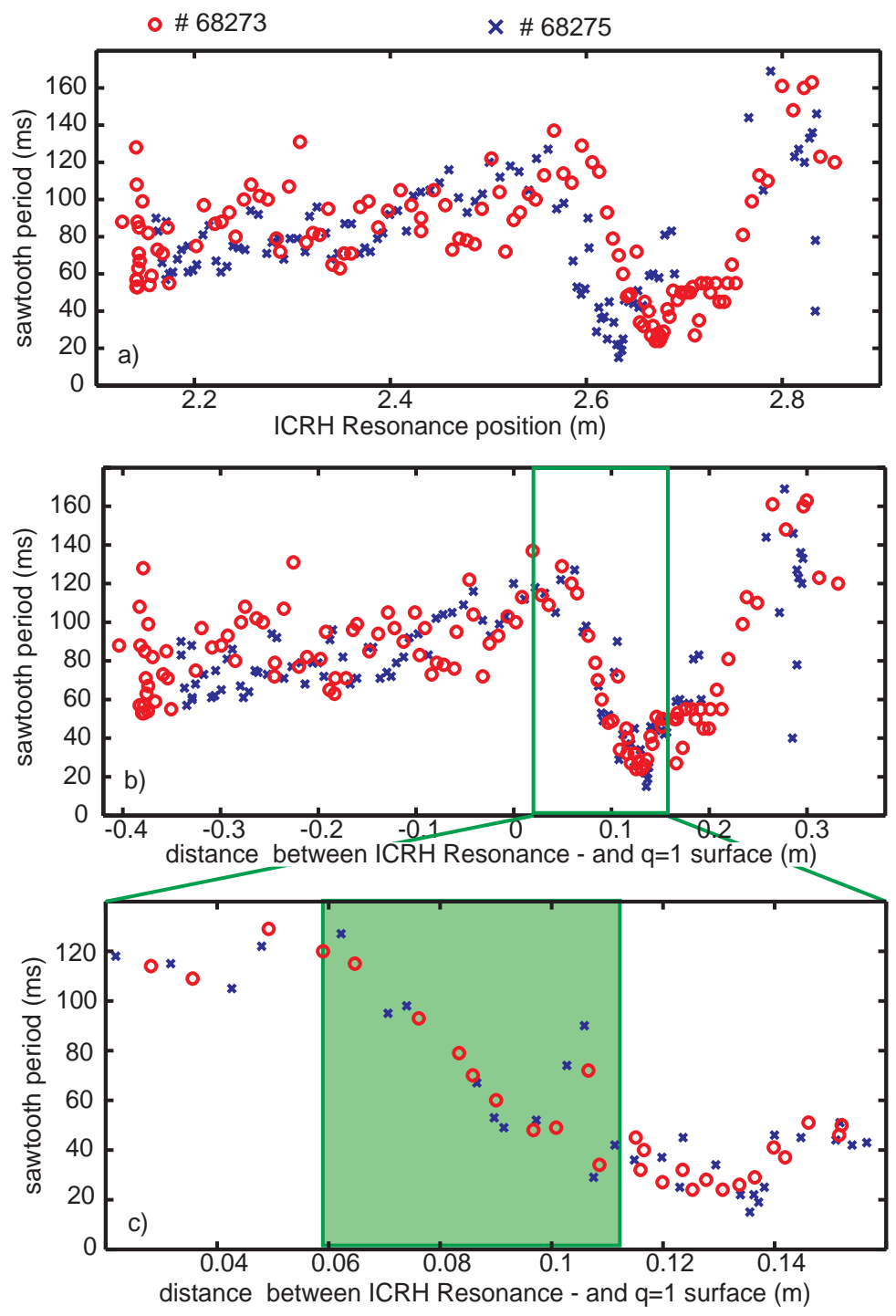

Figure 6: Sawtooth period for the two shots in figure 5: a) as a function of major radius of the ICRF resonance location over the full scan range. b) as a function of the distance between the ICRF resonance and the $\mathrm{q}=1$ position at mid plane (from EFIT) over the full scan range. c) zoom on sawtooth period transition region - the green shaded region shows the range of variation achievable through ICRF real time frequency variation. 


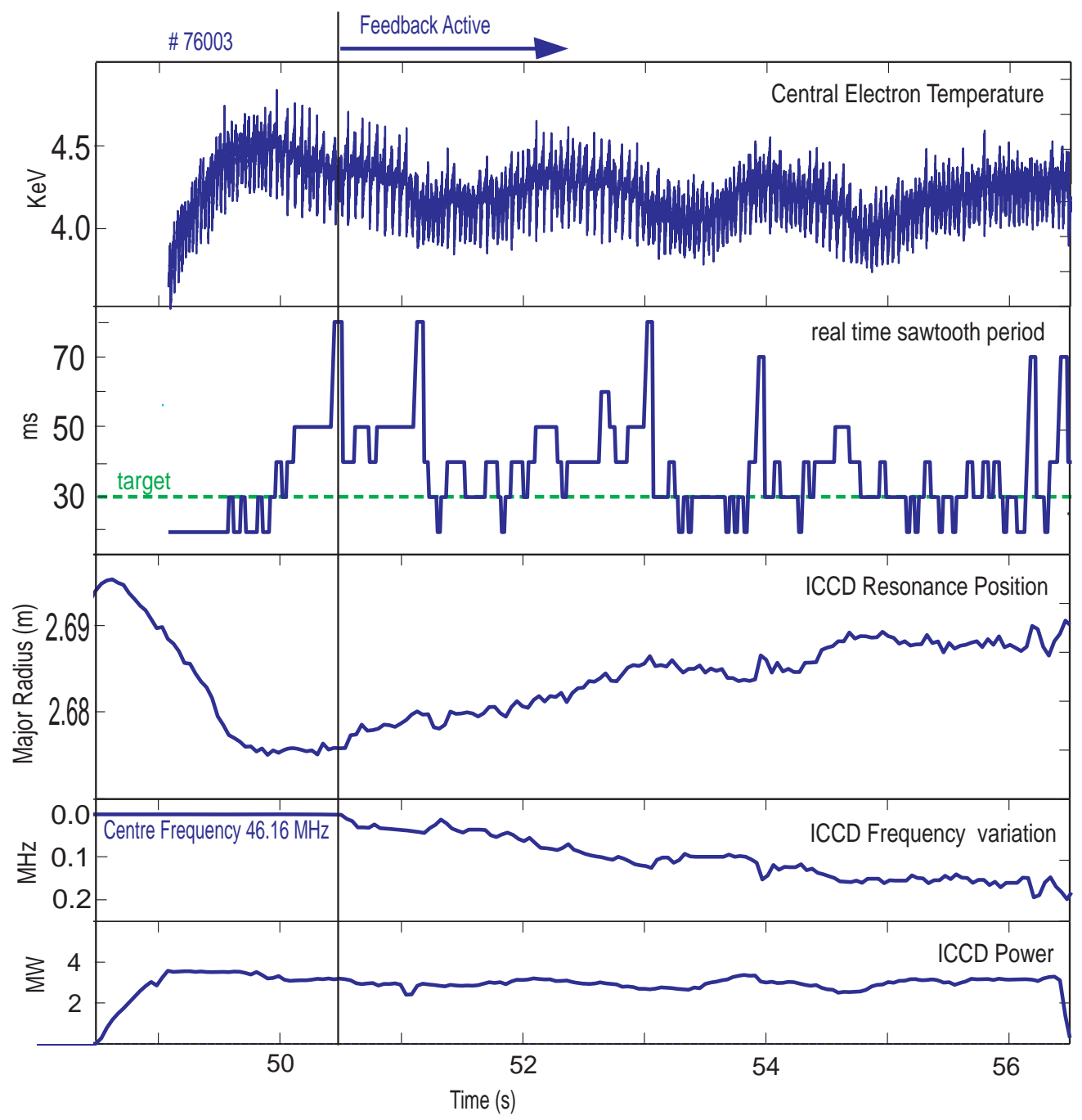

Figure 7 - Successful closed loop sawtooth period control. The target sawtooth period of 30ms is rapidly reached and maintained until the end of the pulse. 


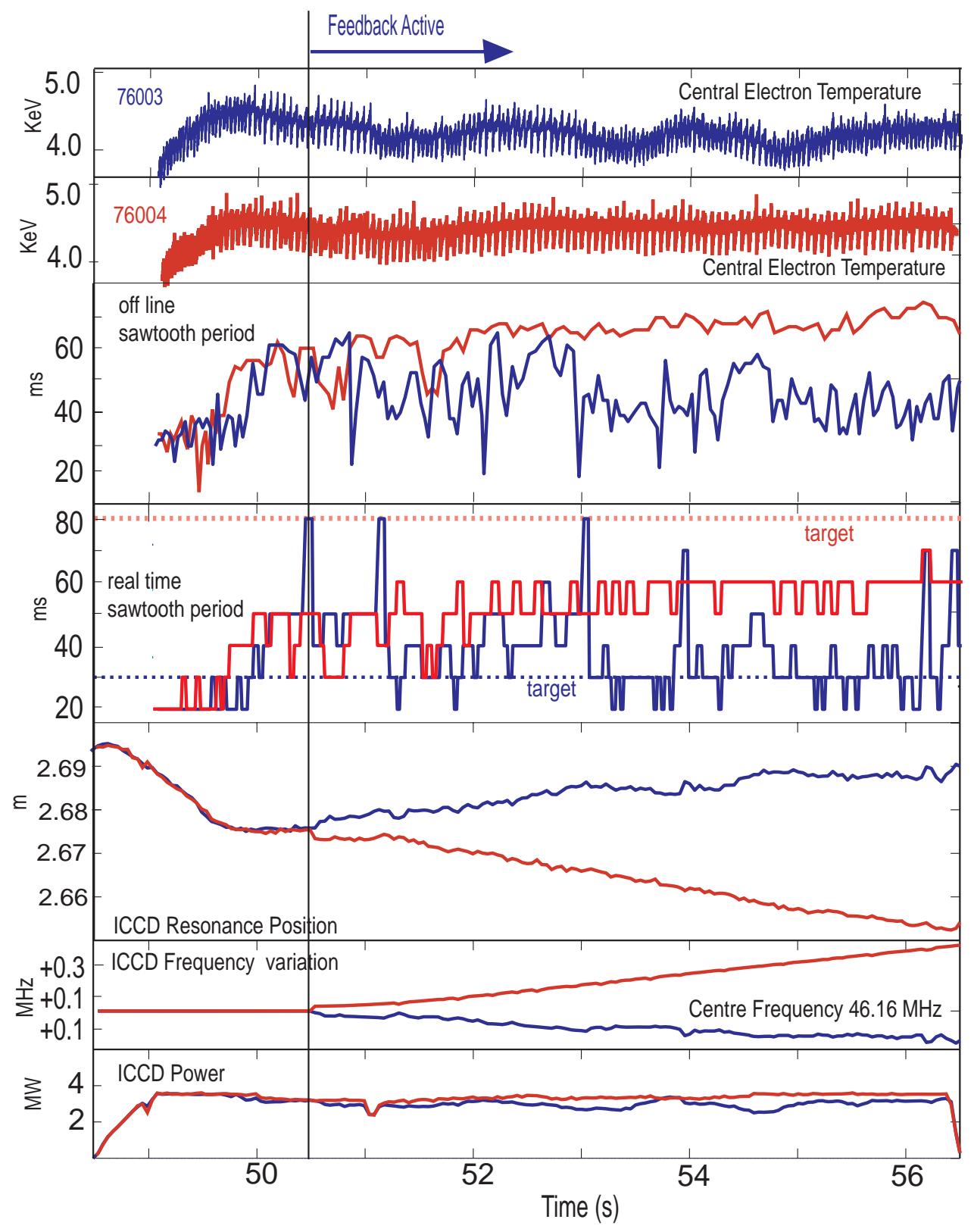

Figure $8-2$ successful discharges with feedback control of the sawtooth period. The first (blue ) is the one shown in figure 7 where the target of $30 \mathrm{~ms}$ is reached, in the second the target was changed to $80 \mathrm{~ms}$. In this case the controller saturated at $60 \mathrm{~ms}$. 


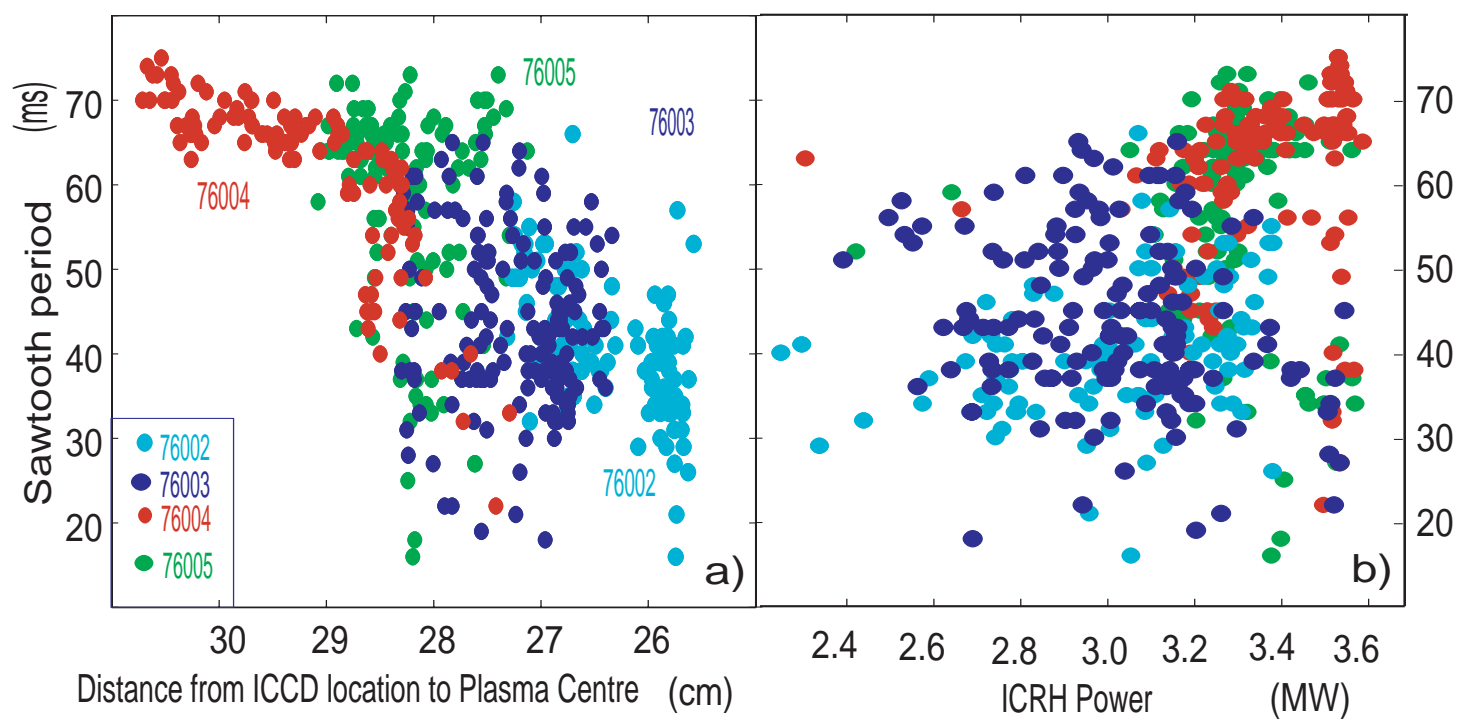

Figure 9 - Sawtooth period as a function of a) ICRF location (distance from the plasma centre) and b) ICRF power. The sawtooth period depends on resonance location rather than the (small) variations in ICRF power. 